\title{
Aproximaciones sobre los discursos y prácticas de la Escuela Activa en Colombia
}

\author{
Luz Andrea Gutiérrez Caro ${ }^{(}$, Liliana Andrea Mariño Díaz \\ Institución Educativa Técnica Miraflores, Universidad Pedagógica y Tecnológica de Colombia - Colombia
}

Autor de correspondencia: 'luzandrea.1213@hotmail.com

Recibido: 28 de febrero de 2020 Revisado: 30 abril de 2020 Aprobado: 30 de julio de 2020 Publicado: 16 de agosto de 2020

\section{Resumen}

Se presenta una construcción teórico-reflexiva como estado del arte de la Escuela Activa en Colombia, el cual hace parte del proyecto Escuela Activa, una experiencia de educación transformadora. Se identifican sus transformaciones, discursos y prácticas, a partir de la revisión de artículos digitales en Publindex. Se definen cinco categorías: configuración teórica del modelo, historia de la Escuela Nueva en Colombia, currículo, metodología y aplicaciones y experiencias, las cuales emergen a partir de una metodología descriptiva, desde una reflexión teórica y la elaboración tanto de una matriz de análisis de datos, como de fichas temáticas - las cuales permitieron hallar otras categorías relevantes y una interrelación entre diversas subcategorías-. Se evidencia la apropiación histórica del modelo, sus efectos positivos, falencias, tendencias, prácticas y vacíos investigativos en torno al programa de Escuela Nueva Activa en el país.

Palabras clave: teoría de la educación, práctica pedagógica, tendencia educacional, Escuela Activa, educación básica

\section{(c) $(1) \Theta$}

Para citar este artículo: Gutierrez, L., \& Mariño, A. (2020). Aproximaciones sobre los discursos y prácticas de la Escuela Activa en Colombia. Praxis \& Saber, 11(27), e10732. https://doi. org/10.19053/22160159.v11.n27.2020.10732 


\title{
An approach to the discourses and practices of the Active School in Colombia
}

\begin{abstract}
A theoretical-reflexive construction is presented as the state of the art of the Active School in Colombia, which is part of the project: Active School, an experience of transformative education. Its transformations, discourses, and practices are identified from the study of digital articles in Publindex. Five categories are defined: Theoretical configuration of the model, History of the New School in Colombia, Curriculum, Methodology and Applications and experiences, which emerge from a descriptive methodology, based on theoretical reflections and on the development of both a matrix for data analysis and subject sheetsthe latter allowed to find other relevant categories and an interrelation between different subcategories. There is evidence of the historical appropriation of the model, its positive effects, flaws, tendencies, practices, and research gaps around the Active New School program in the country.
\end{abstract}

Keywords: educational theory, pedagogical practice, educational tendency, Active School, elementary education

\section{Abordagens aos discursos e práticas da Escola Ativa na Colômbia}

\section{Resumo}

Apresenta-se uma construção teórico-reflexiva como um estado da arte da Escola Ativa na Colômbia, que faz parte do projeto Escola Ativa, uma experiência educacional transformadora. Identificam-se suas transformações, discursos e práticas, com base na revisão de artigos digitais em Publindex. São definidas cinco categorias: configuração teórica do modelo, história da Escola Nova na Colômbia, currículo, metodologia e aplicações e experiências, que emergem de uma metodologia descritiva, a partir de uma reflexão teórica, da elaboração de uma matriz de análise de dados, e da elaboração de folhas temáticas - as quais permitiram encontrar outras categorias relevantes e uma inter-relação entre várias subcategorias. É mostrada uma apropriação histórica do modelo, seus efeitos positivos, deficiências, tendências, práticas e lacunas de investigação em torno do programa Escola Nova Ativa no país.

Palavras-chave: teoria da educação, prática pedagógica, tendência educacional, Escola Ativa, educação básica

La Escuela Activa [EA] hace referencia a un planteamiento pedagógico que infundió ideas de cambio desde principios del s. XX en la educación colombiana. Tiene una trayectoria histórica relativamente amplia en la que ha cimentado sus bases y se ha transformado, especialmente, desde la psicología y la sociología. Sin embargo, su adopción como política 
educativa nacional hace tres décadas llevó a que las comunidades educativas la percibieran como una oportunidad para la escuela unitaria y multigrado rural.

Con la exploración de artículos de revistas electrónicas de educación y pedagogía y con la elaboración de fichas temáticas y analíticas, se pretende identificar la transformación, los discursos y las prácticas de la EA, para conocer su adaptación al contexto nacional en el trasfondo pedagógico cotidiano, sus bases teóricas, su fundamentación histórica y sus principios filosóficos en función del desarrollo político, social y cultural. La minimización del modelo a la aplicación de cartillas ha desdibujado el qué, el cómo, el por qué y el para qué de su apropiación en la educación colombiana, pues no se presentan alternativas para reflexionar sobre los discursos y las prácticas particulares en la educación popular, con miras a comprender maneras diversas de la EA —alejadas de nociones eurocentristas- en las situaciones actuales de Colombia y Latinoamérica (Mejía, 2015).

El documento se divide en tres planos analíticos: (1) en Precauciones metodológicas se muestra un desplazamiento -instrumentos y primeros hallazgos-; (2) Condiciones del discurso es un plano conformado por dos desplazamientos -configuración teórica del modelo pedagógico Escuela Nueva y sujeto e historia de la Escuela Nueva-; y (3) en Prácticas: saber, poder y sujeto se exponen tres desplazamientos - currículo, saber y poder, métodos $y$ relación con el sujeto y Escuela Activa como experiencia-.

\section{Primer plano analítico: precauciones metodológicas}

El estudio parte de una propuesta de investigación cualitativa documental de tipo expositivo de acuerdo con Ramos y Romanowski (2014), quienes afirman que consiste en la formulación histórica del diálogo académico bajo una perspectiva analítica que permite hacer comparaciones, señalar la evolución y los aportes teóricos, comprender los diferentes contextos e identificar tendencias. Se aplicó una metodología descriptiva que tiene en cuenta datos cuantitativos y cualitativos que permiten establecer relaciones entre las categorías y subcategorías emergentes a partir del análisis del contenido para dar respuesta a la siguiente pregunta investigativa: ¿cuáles han sido las trasformaciones, los discursos y las prácticas de Escuela Nueva [EN] / Escuela Activa [EA] en Colombia?

La búsqueda de artículos se centró en revistas electrónicas colombianas de ámbito educativo disponibles en Publindex. El estudio abarca una delimitación temporal desde 1994 hasta 2019, por dos razones: la primera es la experiencia obtenida en cuatro años de trayectoria del modelo a partir de su implementación a través del Decreto 1490 de 1990; la otra razón es el documento Colombia: al filo de la oportunidad (Presidencia de la República - Colciencias, 1995), que habla de la deficiencia de la cobertura, especialmente en la zona rural, que es la principal preocupación del gobierno de Gaviria.

Único desplazamiento: instrumentos y primeros hallazgos

La primera etapa de la metodología de trabajo fue un mapeo bibliográfico general de EA y EN sistematizado en el software Mendeley. Se realizó una matriz general con los datos básicos de cada artículo y una lectura previa para establecer posibles subcategorías como un proceso de conceptualización preliminar (tabla 1). 


\section{Tabla 1}

Matriz general de información

\begin{tabular}{|c|c|c|c|c|c|}
\hline $\mathrm{N}^{\circ}$ & Autor/es & Año & Título & Revista & Institución \\
\hline 1 & Rafael Flórez Ochoa & 1995 & $\begin{array}{l}\text { La dimensión pedagógica, } \\
\text { formación y Escuela Nueva en } \\
\text { Colombia. }\end{array}$ & $\begin{array}{l}\text { Revista de Educación y } \\
\text { Pedagogía }\end{array}$ & $\begin{array}{l}\text { Universidad de } \\
\text { Antioquia [UdeA] }\end{array}$ \\
\hline 2 & $\begin{array}{l}\text { Ernesto Schiefelbein; } \\
\text { Rodrigo Vera; Humberto } \\
\text { Aranda; Zoila Vargas; } \\
\text { Víctor Corco }\end{array}$ & 1996 & $\begin{array}{l}\text { En busca de la escuela del siglo XXI } \\
\text { ¿Puede darnos la pista la Escuela } \\
\text { Nueva de Colombia? }\end{array}$ & $\begin{array}{l}\text { Revista Colombiana de } \\
\text { Educación }\end{array}$ & $\begin{array}{l}\text { Universidad Pedagógica } \\
\text { Nacional [UPN] }\end{array}$ \\
\hline 3 & Rosa María Torres & 1996 & $\begin{array}{l}\text { Alternativas dentro de la educación } \\
\text { formal: el programa de Escuela } \\
\text { Nueva en Colombia }\end{array}$ & $\begin{array}{l}\text { Revista Colombiana de } \\
\text { Educación }\end{array}$ & UPN \\
\hline 4 & $\begin{array}{l}\text { George Psacharopoulos; } \\
\text { Carlos Rojas; Eduardo } \\
\text { Vélez }\end{array}$ & 1996 & $\begin{array}{l}\text { Evaluación de resultados en la } \\
\text { Escuela Nueva de Colombia ¿Es el } \\
\text { multigrado la respuesta? }\end{array}$ & $\begin{array}{l}\text { Revista Colombiana de } \\
\text { Educación }\end{array}$ & UPN \\
\hline 5 & Javier Sáenz Obregón & 1997 & $\begin{array}{l}\text { De lo biológico a lo social. Saber } \\
\text { pedagógico y educación pública en } \\
\text { Colombia. }\end{array}$ & $\begin{array}{l}\text { Revista Educación y } \\
\text { Ciudad }\end{array}$ & $\begin{array}{l}\text { Instituto para la } \\
\text { Investigación Educativa } \\
\text { y el Desarrollo } \\
\text { Pedagógico }\end{array}$ \\
\hline 6 & Raúl Barrantes & 2001 & $\begin{array}{l}\text { Las innovaciones educativas: } \\
\text { escenarios y discursos de una } \\
\text { década en Colombia. }\end{array}$ & Magisterio.com.co & $\begin{array}{l}\text { Sociedad Colombiana } \\
\text { de Pedagogía }[\mathrm{SCP}]\end{array}$ \\
\hline 7 & $\begin{array}{l}\text { Carlos Arturo Londoño } \\
\text { Ramos }\end{array}$ & 2002 & $\begin{array}{l}\text { El pragmatismo de Dewey y la } \\
\text { Escuela Nueva en Colombia }\end{array}$ & $\begin{array}{l}\text { Historia de } \\
\text { la Educación } \\
\text { Colombiana }\end{array}$ & $\begin{array}{l}\text { Universidad de Nariño } \\
\text { [Udenar] }\end{array}$ \\
\hline 8 & Gerardo León Guerrero & 2002 & $\begin{array}{l}\text { La imposición de modelos } \\
\text { pedagógicos en Colombia - Siglo } \\
\text { XX }\end{array}$ & $\begin{array}{l}\text { Revista Estudios } \\
\text { Latinoamericanos }\end{array}$ & Udenar \\
\hline 9 & Fernando Reimers & 2003 & $\begin{array}{l}\text { Aprender a leer y a convivir en las } \\
\text { escuelas rurales de Colombia }\end{array}$ & Pedagogía y Saberes & UPN \\
\hline 10 & $\begin{array}{l}\text { José Edmundo Calvache } \\
\text { López }\end{array}$ & 2003 & $\begin{array}{l}\text { La Escuela Nueva y los conceptos } \\
\text { básicos de la educación en el } \\
\text { pensamiento de John Dewey: Una } \\
\text { aproximación teórica }\end{array}$ & $\begin{array}{l}\text { Revista Historia } \\
\text { de la Educación } \\
\text { Latinoamericana }\end{array}$ & $\begin{array}{l}\text { Universidad Pedagógica } \\
\text { y Tecnológica de } \\
\text { Colombia [UPTC] }\end{array}$ \\
\hline 11 & Néstor Cardoso Erlam & 2003 & $\begin{array}{l}\text { La Escuela Activa y el texto } L a \\
\text { alegría de leer en Colombia }\end{array}$ & $\begin{array}{l}\text { Revista Historia } \\
\text { de la Educación } \\
\text { Latinoamericana }\end{array}$ & UPTC \\
\hline 12 & Vicky Colbert de Arboleda & 2006 & $\begin{array}{l}\text { Mejorar la calidad de la educación } \\
\text { en escuelas de escasos recursos. } \\
\text { El caso de la Escuela Nueva en } \\
\text { Colombia }\end{array}$ & $\begin{array}{l}\text { Revista Colombiana de } \\
\text { Educación }\end{array}$ & UPN \\
\hline 13 & $\begin{array}{l}\text { Lucero Ruiz Jiménez; } \\
\text { Jaime Alberto Pineda } \\
\text { Muñoz }\end{array}$ & 2007 & $\begin{array}{l}\text { Hacia una fundamentación } \\
\text { epistemológica del modelo de } \\
\text { pedagogía activa }\end{array}$ & Plumilla Educativa & $\begin{array}{l}\text { Universidad de } \\
\text { Manizales [Umanizales] }\end{array}$ \\
\hline 14 & Yolanda Rojas Galvis & 2007 & $\begin{array}{l}\text { Escuela Activa Urbana. Un proyecto } \\
\text { sostenible }\end{array}$ & Plumilla Educativa & Umanizales \\
\hline 15 & $\begin{array}{l}\text { Ana María González De } \\
\text { Londoño; Santiago Isaza } \\
\text { Arango }\end{array}$ & 2007 & Escuela Activa Urbana & Plumilla Educativa & Umanizales \\
\hline 16 & María Aracelly López Gil & 2007 & $\begin{array}{l}\text { Aportes de la pedagogía activa a la } \\
\text { educación }\end{array}$ & Plumilla Educativa & Umanizales \\
\hline 17 & Odilia Ramírez Contreras & 2008 & $\begin{array}{l}\text { The English Language Learning } \\
\text { inside the Escuela Activa Urbana } \\
\text { Model in a public School. A study of } \\
\text { sixth graders }\end{array}$ & Profile & $\begin{array}{l}\text { Universidad Nacional } \\
\text { de Colombia [Unal] }\end{array}$ \\
\hline
\end{tabular}




\begin{tabular}{|c|c|c|c|c|c|}
\hline 18 & $\begin{array}{l}\text { Florentino Rico Calvano; } \\
\text { Juan Spirko Payares }\end{array}$ & 2008 & Crónica sobre el Gimnasio Moderno & $\begin{array}{l}\text { Educación y } \\
\text { Humanismo }\end{array}$ & $\begin{array}{l}\text { Universidad Simón } \\
\text { Bolívar }\end{array}$ \\
\hline 19 & $\begin{array}{l}\text { Bernardo Barragán } \\
\text { Castrillón }\end{array}$ & 2008 & $\begin{array}{l}\text { María Montessori: los límites } \\
\text { de la escuela activa, una lectura } \\
\text { arqueológica }\end{array}$ & Nodos y Nudos & UPN \\
\hline 20 & $\begin{array}{l}\text { Ángela María Jiménez } \\
\text { Avilés }\end{array}$ & 2009 & $\begin{array}{l}\text { La Escuela Nueva y los espacios } \\
\text { para educar }\end{array}$ & $\begin{array}{l}\text { Revista Educación y } \\
\text { Pedagogía }\end{array}$ & UdeA \\
\hline 21 & Rodrigo Villar & 2010 & $\begin{array}{l}\text { El programa de Escuela Nueva en } \\
\text { Colombia }\end{array}$ & $\begin{array}{l}\text { Revista Educación y } \\
\text { Pedagogía }\end{array}$ & UdeA \\
\hline 22 & $\begin{array}{l}\text { Camilo Andrés Ramírez } \\
\text { López; Henry Portela } \\
\text { Guarín; Luis Hernando } \\
\text { Amador Pineda }\end{array}$ & 2010 & $\begin{array}{l}\text { Actitud y valores en la clase de } \\
\text { educación física. Un caso desde el } \\
\text { modelo Escuela Nueva }\end{array}$ & $\begin{array}{l}\text { Revista de } \\
\text { Investigaciones }\end{array}$ & $\begin{array}{l}\text { Universidad Católica de } \\
\text { Manizales }\end{array}$ \\
\hline 23 & Víctor Manuel Gómez & 2010 & $\begin{array}{l}\text { Visión crítica sobre la Escuela } \\
\text { Nueva en Colombia }\end{array}$ & $\begin{array}{l}\text { Revista de Educación y } \\
\text { Pedagogía }\end{array}$ & UdeA \\
\hline 24 & $\begin{array}{l}\text { Ómar Armando Villota } \\
\text { Pantoja }\end{array}$ & 2011 & La vieja Escuela Nueva & Revista Unimar & $\begin{array}{l}\text { Universidad Mariana } \\
\text { [Umariana] }\end{array}$ \\
\hline 25 & Andrea Guerrero Mosquera & 2011 & $\begin{array}{l}\text { Don Jorge Buendía, el impulsor } \\
\text { de la Escuela Activa en el sur de } \\
\text { Colombia durante el siglo XX }\end{array}$ & $\begin{array}{l}\text { Historia de } \\
\text { la Educación } \\
\text { Colombiana }\end{array}$ & Udenar \\
\hline 26 & $\begin{array}{l}\text { Andrés Klaus Runge Peña; } \\
\text { Diego Alejandro Muñoz } \\
\text { Gaviria }\end{array}$ & 2011 & $\begin{array}{l}\text { Actividad vs. agitación en el } \\
\text { pensamiento de Luis López de } \\
\text { Mesa: relaciones entre pedagogía } \\
\text { y eugenesia en la Colombia de } \\
\text { principios del siglo XX }\end{array}$ & $\begin{array}{l}\text { Revista Colombiana de } \\
\text { Educación }\end{array}$ & UPN \\
\hline 27 & $\begin{array}{l}\text { Camilo Andrés Ramírez } \\
\text { López; William Rolando } \\
\text { Ardila Rodríguez; Nelson } \\
\text { Hernando Montaño } \\
\text { Fandiño }\end{array}$ & 2013 & $\begin{array}{l}\text { Tareas motrices. Apuesta } \\
\text { comprensiva para su diseño } \\
\text { metodológico en Escuela Nueva }\end{array}$ & Impetus & $\begin{array}{l}\text { Universidad de los } \\
\text { Llanos }\end{array}$ \\
\hline 28 & $\begin{array}{l}\text { Mauro Andrés Imbachí } \\
\text { Polo }\end{array}$ & 2013 & $\begin{array}{l}\text { La Escuela Nueva y las } \\
\text { competencias lectoras desarrolladas } \\
\text { en el área de lengua castellana }\end{array}$ & $\begin{array}{l}\text { Revista Digital de } \\
\text { Ciencias Aplicadas al } \\
\text { Deporte }\end{array}$ & Universidad del Tolima \\
\hline 29 & Rafael Ríos Beltrán & 2013 & $\begin{array}{l}\text { Escuela Nueva y saber pedagógico } \\
\text { en Colombia: apropiación, } \\
\text { modernidad y métodos de } \\
\text { enseñanza. Primera mitad del siglo } \\
\text { XIX }\end{array}$ & Historia y Sociedad & Unal \\
\hline 30 & $\begin{array}{l}\text { Nubia Elena Pineda de } \\
\text { Cuadros }\end{array}$ & 2014 & $\begin{array}{l}\text { Reflexiones sobre la labor docente } \\
\text { en escuelas rurales que implementan } \\
\text { la metodología Escuela Nueva }\end{array}$ & $\begin{array}{l}\text { Quaestiones } \\
\text { Disputatae: Temas en } \\
\text { Debate }\end{array}$ & $\begin{array}{l}\text { Universidad Santo } \\
\text { Tomás [USTA] }\end{array}$ \\
\hline 31 & Napoleón Murcia Peña & 2014 & $\begin{array}{l}\text { Comunidades de práctica: huellas } \\
\text { en el currículo de la Escuela Activa } \\
\text { Urbana en Manizales }\end{array}$ & Criterios & Umariana \\
\hline 32 & $\begin{array}{l}\text { Rafael Ríos Beltrán; } \\
\text { Martha Yanet Cerquera } \\
\text { Cuellar }\end{array}$ & 2014 & $\begin{array}{l}\text { La modernización de los contenidos } \\
\text { y métodos de enseñanza: reflexiones } \\
\text { sobre Escuela Nueva en Colombia }\end{array}$ & $\begin{array}{l}\text { Revista Historia } \\
\text { de la Educación } \\
\text { Latinoamericana }\end{array}$ & UPTC \\
\hline 33 & Ángel Marcel & 2014 & $\begin{array}{l}\text { Don Agustín Nieto Caballero, } \\
\text { andante de la educación }\end{array}$ & $\begin{array}{l}\text { Revista Historia } \\
\text { de la Educación } \\
\text { Latinoamericana }\end{array}$ & UPTC \\
\hline 34 & $\begin{array}{l}\text { María Janet Barreto } \\
\text { Morales; Myriam } \\
\text { Leguizamón Roa; Jaime } \\
\text { Andrés Torres Ortiz }\end{array}$ & 2015 & $\begin{array}{l}\text { La comprensión lectora a través de } \\
\text { las guías de aprendizaje en Escuela } \\
\text { Nueva }\end{array}$ & Educación y Ciencia & UPTC \\
\hline 35 & $\begin{array}{l}\text { Armando González } \\
\text { Gutiérrez; Mónica } \\
\text { Jacqueline Regalado } \\
\text { Cañón; Alfonso Jiménez } \\
\text { Espinosa }\end{array}$ & 2015 & $\begin{array}{l}\text { La pedagogía activa con } \\
\text { metodología Escuela Nueva en } \\
\text { Boyacá: el caso de dos municipios }\end{array}$ & $\begin{array}{l}\text { Quaestiones } \\
\text { Disputatae: Temas en } \\
\text { Debate }\end{array}$ & USTA \\
\hline
\end{tabular}




\begin{tabular}{|c|c|c|c|c|c|}
\hline 36 & Jaime Cuadros Dávila & 2015 & $\begin{array}{l}\text { Escuela Nueva: una estrategia } \\
\text { pedagógica en la Institución } \\
\text { Educativa Agropecuaria Santa } \\
\text { Bárbara }\end{array}$ & $\begin{array}{l}\text { Revista Historia } \\
\text { de la Educación } \\
\text { Colombiana }\end{array}$ & Udenar \\
\hline 37 & $\begin{array}{l}\text { Diana Carolina Suárez } \\
\text { Díaz; Andrea del Pilar Liz; } \\
\text { Carlos Fernando Parra } \\
\text { Moreno }\end{array}$ & 2015 & $\begin{array}{l}\text { Construyendo tejido social desde } \\
\text { la Escuela Nueva en Colombia. Un } \\
\text { estudio de caso. }\end{array}$ & $\begin{array}{l}\text { Revista Científica José } \\
\text { Maria Córdova }\end{array}$ & $\begin{array}{l}\text { Escuela Militar de } \\
\text { Cadetes General José } \\
\text { María Córdova }\end{array}$ \\
\hline 38 & $\begin{array}{l}\text { Vilma Cristina Sánchez } \\
\text { Macías; Yolanda Ibeth } \\
\text { Amado Sarmiento; } \\
\text { Alejandro Bolívar Suárez }\end{array}$ & 2015 & $\begin{array}{l}\text { Enseñanza de lectura literal } \\
\text { en resolución de problemas } \\
\text { matemáticos en Escuela Nueva }\end{array}$ & Educación y Ciencia & UPTC \\
\hline 39 & $\begin{array}{l}\text { Katherine Redondo de Oro; } \\
\text { César Redondo Bermúdez; } \\
\text { Víctor Martínez Saravia }\end{array}$ & 2015 & $\begin{array}{l}\text { Aportes del proyecto 'Escuela } \\
\text { Nueva' a la educación universitaria }\end{array}$ & $\begin{array}{l}\text { Revista Ciencias } \\
\text { Biomédicas }\end{array}$ & $\begin{array}{l}\text { Universidad de } \\
\text { Cartagena }\end{array}$ \\
\hline 40 & Nelson Torres Vega & 2015 & $\begin{array}{l}\text { Agustín Nieto Caballero: } \\
\text { pensamiento pedagógico y aportes a } \\
\text { la Escuela Nueva }\end{array}$ & Revista Unimar & Umariana \\
\hline 41 & $\begin{array}{l}\text { Nilza Offrir García Vera; } \\
\text { Sandra Lucía Rojas Prieto }\end{array}$ & 2015 & $\begin{array}{l}\text { La enseñanza de la lectura en } \\
\text { Colombia: enfoques pedagógicos, } \\
\text { métodos, políticas y textos escolares } \\
\text { en las tres últimas décadas del } \\
\text { siglo XX }\end{array}$ & $\begin{array}{l}\text { Revista Pedagogía y } \\
\text { Saberes }\end{array}$ & UPN \\
\hline 42 & $\begin{array}{l}\text { Carlos Julio Vivas Silva; } \\
\text { Zulma Lucía Murillo } \\
\text { Chiquillo; Jaime Ricardo } \\
\text { Cristancho Chinome }\end{array}$ & 2016 & $\begin{array}{l}\text { Scratch. Estrategia didáctica para } \\
\text { el aprendizaje de las tablas de } \\
\text { multiplicar en Escuela Nueva }\end{array}$ & Educación y Ciencia & UPTC \\
\hline 43 & Absalón Jiménez Becerra & 2017 & $\begin{array}{l}\text { Primer Congreso Pedagógico } \\
\text { Nacional Colombiano de } 1917\end{array}$ & Pedagogía y Saberes & UPN \\
\hline 44 & Mary Luz Bernal-Pinzón & 2017 & $\begin{array}{l}\text { ¿Qué escriben los niños? Una } \\
\text { mirada desde el modelo Escuela } \\
\text { Nueva }\end{array}$ & $\begin{array}{l}\text { Revista de } \\
\text { Investigación, } \\
\text { Desarrollo e } \\
\text { Innovación }\end{array}$ & UPTC \\
\hline 45 & $\begin{array}{l}\text { Nidia Yaneth Torres } \\
\text { Merchán; Marisol Rojas } \\
\text { Niño }\end{array}$ & 2017 & $\begin{array}{l}\text { Enseñanza de especies no } \\
\text { carismáticas invertebradas en el } \\
\text { contexto de la Escuela Nueva: un } \\
\text { análisis desde los libros de texto }\end{array}$ & Bio-grafia & UPN \\
\hline 46 & Sara Evelin Urrea Quintero & 2017 & $\begin{array}{l}\text { Las agencias internacionales, su } \\
\text { papel en la creación y expansión del } \\
\text { modelo Escuela Nueva }\end{array}$ & Ágora USB & $\begin{array}{l}\text { Universidad de San } \\
\text { Buenaventura }\end{array}$ \\
\hline 47 & $\begin{array}{l}\text { Hugo Alfonso Ávila } \\
\text { Cepeda; Rosalbina Jiménez } \\
\text { Pinzón; Jaime Andrés } \\
\text { Torres Ortiz }\end{array}$ & 2017 & $\begin{array}{l}\text { Comprensión lectora en estudiantes } \\
\text { de primero a tercero de Escuela } \\
\text { Nueva. }\end{array}$ & Educación y Ciencia & UPTC \\
\hline 48 & Julián de Zubiría Samper & 2019 & $\begin{array}{l}\text { ¿Favorece la Escuela Nueva } \\
\text { el desarrollo del interés por el } \\
\text { conocimiento? }\end{array}$ & Revista Magisterio & SCP \\
\hline 49 & Julián de Zubiría Samper & 2019 & 4 aportes de la Escuela Nueva & Revista Magisterio & SCP \\
\hline 50 & Fundación Escuela Nueva & 2019 & $\begin{array}{l}\text { Cooperative Learning in Escuela } \\
\text { Nueva Activa }\end{array}$ & Revista Magisterio & SCP \\
\hline 51 & Fundación Escuela Nueva & 2019 & $\begin{array}{l}\text { La Educación para la Paz que } \\
\text { Promueve la Fundación Escuela } \\
\text { Nueva Volvamos a la Gente }\end{array}$ & Revista Magisterio & SCP \\
\hline 52 & Julián de Zubiría Samper & 2019 & $\begin{array}{l}\text { Escuela Nueva: ¿Desarrolla el } \\
\text { activismo la flexibilidad? }\end{array}$ & Revista Magisterio & SCP \\
\hline
\end{tabular}

En las revistas digitales se encontraron 52 artículos. Se evidenció que hubo mayor productividad académica en 2015 en torno a adaptaciones y estrategias pedagógicas sugeridas a procesos de enseñanza-aprendizaje y al desarrollo de competencias sociales, 
emergentes de los principios de la pedagogía activa y recursos de la EN. Allí se resaltan falencias del modelo en dichos procesos y se invita a proponer mejoras y actualizaciones del material de apoyo. Otro factor de aumento en las publicaciones es el aumento de investigaciones sobre este tema entre 2017 y 2019.

Las publicaciones de la UPN en su mayoría exponen estudios relacionados con los fundamentos teóricos y la historia de la EN. En la UPTC se hallaron textos enfocados en la construcción y aplicación de estrategias de lectura, de escritura y de matemáticas.

Las publicaciones de la revista Magisterio de la Sociedad Colombiana de Pedagogía muestran una marcada tendencia hacia el estudio de los efectos y aportes de la EN en cuanto al trabajo de habilidades cognitivas y competencias sociales.

Finalmente, se recolectó y se organizó la información en fichas temáticas para identificar los fundamentos pedagógicos y abarcar las reformas educativas, adaptaciones y vacíos del modelo. Las fichas dieron pautas para señalar elementos fundamentales en el análisis propuesto, a partir del registro de las palabras clave y resumen de cada uno. Este proceso condujo a la identificación de enfoques dominantes y a la posterior consolidación de categorías y subcategorías.

La tabla 2 evidencia las categorías generales de la EN que hacen referencia con mayor frecuencia a modelo pedagógico y currículo, mientras las menos publicadas se relacionan con el tema de historia.

\section{Tabla 2}

Dominancia de categorías

\begin{tabular}{lll}
\hline Categoría & $\mathbf{N}^{\circ}$ & $\mathbf{\%}$ \\
\hline Currículo de la Escuela Nueva & 56 & 29.48 \\
Modelo Pedagógico Escuela Nueva & 54 & 28.42 \\
Metodología de Escuela Nueva & 50 & 26.31 \\
Aplicaciones y experiencias & 20 & 10.52 \\
Historia de la Escuela Nueva en Colombia & 10 & 5.27 \\
\hline Total general & 190 & 100 \\
\hline
\end{tabular}

\section{Segundo plano analítico: condiciones del discurso}

El presente plano tiene como objetivo evidenciar los enunciados que provienen de la EA y que hacen parte del mismo orden epistemológico. Estos no se conciben como enunciados separados, sino que se entrelazan a causa del análisis de un discurso que evidencia categorías, subcategorías y nociones que resultan del estado del arte.

Primer desplazamiento: configuración teórica del modelo pedagógico de Escuela Nueva

Abordar los discursos y prácticas implica definir el modelo, comprender sus principios y establecer su relación con la educación colombiana. La EN es una alternativa moderna frente al pensamiento científico, la ciudadanía, la tecnología y la burocracia nacional que fundamenta el saber en la actividad y la racionalidad científica en el desarrollo de proyectos (Londoño, 2002); por su parte, Cuadros (2014) hace referencia a que la libertad, el respeto 
por los intereses, las necesidades y la autonomía del niño en el desarrollo de sus habilidades, el pensamiento crítico y el trabajo colaborativo hacen parte de dicha fundamentación.

La participación de diversos campos de conocimiento en la constucción teórica de EA simbolizó la revolución educativa a finales del siglo XIX, gracias a los aportes de Dewey y Claparéde frente a la actividad experimental en los espacios de aprendizaje, la educación mental, los centros de interés, los proyectos, la investigación y la exposición; así como a la inclinación por la primera infancia, los juguetes educativos, las actividades lúdicas y los grupos cooperativos de Froebel, Pestalozzi, Kershensteiner y Freinet (Calvache, 2003).

El abordaje de ciencias experimentales para el sustento teórico de EA tiene su origen en el pensamiento estadounidense y el europeo, fundamentados en la obra de Herbart, Decroly, Claparéde, Montessori, Dewey y Piaget. Se destacan el método global de lectura, de cálculo y los centros de interés de Decroly mediados por la cognición, la dimensión intrínseca y su aplicación social (García \& Rojas, 2015; López, 2007).

La nueva pedagogía se inclina por la enseñanza práctica para corregir la debilidad física y moral de la raza latina en busca de la mejora de las condiciones sociales, culturales y económicas como símbolo de progreso nacional (León, 2002; Runge \& Muñoz, 2011). El componente intrínseco del ser ocupa un espacio predominante en la filosofía de la EN basada en el modelo ilustrado de Kant, la cual privilegia los principios espirituales y morales en la formación de la persona (Rico \& Spirko, 2008).

El interés por la construcción de una sociedad enmarcada en los valores y en la moral llevó a que dichos discursos tocaran contextos educativos con condiciones sociales particulares bajo la filosofía constructivista. En este sentido Schiefelbein (1993) se refiere a la EN como una experiencia de avance educativo acorde con la globalización, los retos actuales, el bilingüismo y la inclusión de la informática. No obstante, el debate encaminado a comprender la coherencia entre el discurso y la realidad de la EN, es clave para determinar la validez de las innovaciones pedagógicas que se le ameritan (Barrantes, 2001).

Un autor que da muestra de ello es Flórez (1995), quien afirma que un avance educativo de la EN fue identificar al niño como un ser con necesidades, intereses y talentos que se forman a partir de un proceso interior y progresivo a través de su participación, definición, convivencia y experiencias. Para Zubiría (2019b), la infancia es la etapa más importante en el desarrollo humano. Las capacidades del niño están relacionadas con el desarrollo de su psicología genética; elemento que hace parte del discurso biológico y que es usado para identificar las aptitudes y el nivel de inteligencia de los estudiantes con el fin de establecer las bases sobre las que la escuela trabajará en pro de la regeneración moral y social de la raza desde la individualidad del ser (Saénz, 1997).

Al respecto, Barragán (2008) y Bernal-Pinzón (2017) resaltan que la escuela de Montessori establece una estrecha relación entre la actividad, el determinismo biológico y el ambiente, donde el docente asume el rol de guía y da protagonismo al aprendizaje como ejercicio de libertad en el que se reconocen los intereses cognitivos del niño. Para Marcel (2003), la apropiación del método en el preescolar se orienta a la espontaneidad del niño, a la acción didáctica del maestro y a la innovación de materiales educativos. Sin embargo, Zubiría (2019a) en este postulado señala un error, que consiste en la falta de atención a la diferencia entre las motivaciones infantiles y los intereses cognitivos, por lo que se requiere la intervención del adulto durante el proceso. 
Para Rousseau el niño ve, piensa y siente diferente según su etapa biológica, lo cual facilita la pedagogía científica. De igual forma, Froebel consideró que la escuela debe adaptarse al niño y utilizar su actividad para la educación (López,2007).

Desde esta perspectiva, González et al. (2015) identifican tres aspectos fundamentales: la libertad, la atención a problemas sociales y el aprender haciendo - este último relacionado con los espacios de aprendizaje-. En este sentido, Guerrero (2011) señala que las excursiones pedagógicas y la enseñanza al aire libre hicieron parte del papel innovador de Jorge Buendía Narváez en la Escuela Normal de Nariño. Esto exigió la formación de un maestro especializado con amplio conocimiento cultural, responsable, amante de la infancia y capaz de generar autoridad, elementos que caracterizaron al Gimnasio Moderno como primer laboratorio pedagógico del país (Torres, 2015).

De acuerdo con Murcia (2014), los intereses sociales y políticos de la pedagogía activa en Colombia y el protagonismo del periodo liberal bajo los postulados del constructivismo y del aprendizaje colaborativo hicieron parte de la respuesta a la anhelada democracia republicana, social y participativa.

Actualmente la transformación de espacios de aprendizaje de contextos educativos vulnerables se complementa, según Villota(2011), con la pedagogía del amor, orientada a la libertad y la justicia, caso específico de los círculos de aprendizaje en poblaciones víctimas del conflicto.

Gracias al recorrido histórico y al papel transformador de la EN, el programa se convirtió en referente de innovación en cuanto a currículo, capacitación, administración y relaciones con la comunidad (Cuadros, 2014; Suárez et al., 2015; Villar, 2010).

Segundo desplazamiento: sujeto e historia de la Escuela Nueva

Los intereses políticos y sociales que permearon el discurso pedagógico a finales del siglo XIX se debieron a las necesidades de la nación, al abordaje de ideas de cambio y a las nuevas formas de orden social. Para Jiménez (2009) la EN nace de los principios de libertad e igualdad promulgados durante la Ilustración y la Revolución francesa. Flórez (1995) y Pineda (2014) catalogan estos valores como insignia de renovación pedagógica. Sin embargo, su posicionamiento en Colombia fue lento y con altibajos por la discrepancia entre los partidos políticos de la época. Sus bases se cimentan en los años 70, en una alternativa educativa conocida como Escuela Progresista, expandida a partir de escuelas experimentales en Antioquia como esperanza de modernización y de progreso social (Runge \& Muñoz, 2011).

La urgencia por atender problemas sociales de amplio impacto para el progreso nacional empezó a solventarse con la socialización de la Ley 39 de 1903, en el primer Congreso Pedagógico Nacional por parte de José Uribe Gaviria. Sus resultados se vieron reflejados en la consolidación de la Universidad Pedagógica Nacional y de dos normales por departamento, en las misiones pedagógicas que promovieron la necesidad de contratar maestros extranjeros - Ley 12 de 1890-, en la implementación de la enseñanza agrícola Ley 38 de 1914- y en la creación de institutos pedagógicos nacionales — Ley 25 de 1917(Torres, 2015; Jiménez, 2018).

La búsqueda de mecanismos que permitieran mitigar los efectos de la Guerra de los mil 
días llevó a la transformación de las costumbres a través de la educación. El texto La alegría de leer de Evangelista Quintana se acopló a los intereses modernizantes y a la reducción del analfabetismo entre los años 30 y los 60 (Cardoso, 2003; Londoño, 2002).

Según López (2007), Nieto Caballero se apropió del nuevo discurso pedagógico y promovió la extensión de la pedagogía activa a primaria, bachillerato y universidades . Asimismo, promovió la renovación de las escuelas normales como centros de investigación y especialización docente y científica. No obstante, Torres (2015) menciona el gran obstáculo que fue la insuficiencia de maestros capacitados para asumir tal reto. Este fue el motivo de las reformas de las instituciones formadoras de docentes - Decreto 10 de 1932-. Por su parte, Ríos (2013) señala que se presentaron resistencias y limitaciones políticas, culturales y metodológicas. Además, Sáenz (1997) señala que la decadencia de las reformas educativas logradas fue causada por la censura eclesiástica y el regreso del partido conservador al poder —debido a sus campañas en contra de la EA-; lo que llevó según León (2002), a la finalización de programas de innovación educativa y la recuperación del modelo pedagógico tradicional.

Según Gómez (2010), Cuádros (2015) y Urrea (2017), en 1961 se retomó la pedagogía activa en la escuela unitaria rural con estrategias multigrado, aprendizaje activo, promoción flexible y recursos de autoinstrucción, lo cual fue un aporte del gobierno de Alberto Lleras Camargo para solventar las problemáticas educativas en ese contexto. Pamplona Santander-fue foco experimentaly, gracias al Banco Interamericano, la EN logró expandirse. En 1987, el Ministerio de Educación Nacional [MEN], con el plan de universalización, dotó de material a las escuelas rurales del país y proporcionó capacitación a sus docentes. Hacia el año 2000 se realizó la misma acción a través del proyecto de educación rural (Imbachí, 2013).

La implementación de métodos activos fue apoyada por la Unesco con el proyecto de enseñanza multigrado, promovido por escuelas normales, la Federación de Cafeteros, la Universidad de Antioquia y la Fundación de Educación Superior (Schiefelbein et al., 1996; Urrea, 2017; Villar, 2010).

El nacimiento de la EN como fundación se debió a problemas con la escuela multigrado. Desde esta perspectiva, la fundación de Colbert contribuyó al mejoramiento de cobertura y finalización de la primaria, lo cual se convirtió en política educativa durante el gobierno de Belisario Betancurt (Cuadros, 2015).

El proyecto iniciado en Norte de Santander, Boyacá y Cundinamarca es asumido por Villar (2010) como una estrategia para lograr la universalización rural y para brindar acceso, retención y calidad. Sin embargo, Redondo et al. (2015) suman a la discusión problemas de deserción, falta de motivación, desigualdad, violencia y abandono de comunidades por parte del gobierno, lo que pone en tela de juicio sus intereses progresistas. De acuerdo con Torres (1996), la relación entre la EN y el MEN está precedida por conflictos que debilitan su expansión. En consecuencia, la fundación ha tenido que recurrir al respaldo de organizaciones privadas, lo que muestra que el apoyo del gobierno central y de la sociedad civil es un factor determinante en la calidad, la responsabilidad, la transparencia y la confianza que se requieren para potenciar innovaciones pedagógicas (Reimers, 2003).

Para Colbert (2006), el modelo ha presentado falencias de gestión y de administración 
que asumió y abordó con la organización de dos congresos internacionales de EN, de los que resultó su expansión a otros contextos, por lo que a partir de 1988 se denominó Escuela Nueva Activa.

La reunión de esfuerzos de la Fundación Escuela Nueva [FEN] por materializar la pedagogía activa en contextos vulnerables se refleja en los reconocimientos obtenidos por parte de instituciones internacionales. La FEN (2019a) menciona uno hecho por Unicef, en el que asume el modelo como una oportunidad de educación para la paz, porque permite la construcción de la autoestima, la cooperación, el trabajo en comunidad y el comportamiento cívico. González et al. (2015) mencionan el premio WISE Prize del 2013, por el impacto producido en los establecimientos educativos de escasos recursos.

El efecto de la EN está relacionado con la búsqueda de la igualdad de oportunidades y con la esperanza en poblaciones vulnerables. A pesar de esto, Psacharopoulos et al. (1996) consideran que resulta complejo mantener la calidad inicial, debido a su alto índice de expansión. En consecuencia, es necesario investigar de qué manera la EN es un modelo que permite aumentar la calidad mientras asume los retos del siglo XXI (Schiefelbein et al., 1996).

\section{Tercer plano analítico: prácticas — saber, poder y sujeto-}

Este apartado está conformado por tres desplazamientos que presentan la práctica como un dispositivo de análisis en las experiencias de EN que están atravesadas por el saber, el poder y el sujeto, en correlación con los discursos.

Primer desplazamiento: currículo, saber y poder

El currículo de la EN se aborda como un conjunto de contenidos plasmados en las guías de aprendizaje, que priorizan la práctica, la experiencia y el entorno para potenciar la productividad y el trabajo (Urrea, 2017).

Para Zubiría (2019c), la flexibilidad del modelo comprende el valor de la individualidad, el rol del docente, la organización física del aula y la importancia de la infancia, elementos que dejan en evidencia la búsqueda de la formación de la opinión, de la espontaneidad y de la mentalidad abierta a la diversidad y al pluralismo. El modelo respeta el ritmo de aprendizaje, se ajusta a las necesidades del estudiante, quien no pierde el año y puede ser promovido en cualquier época del año como una oportunidad para culminar la primaria (Villar, 2010).

Hablar de estrategias de autoaprendizaje implica remitirse a las guías de trabajo. Pineda (2014) resalta que son construidas con base en los principios pedagógicos de Vygotski; promueven la reflexión, la colaboración, el diálogo, la participación y la construcción social de conocimientos. Para Schiefelbein (1993) tienen en cuenta los conocimientos previos, la observación y la sistematización de experiencias.

Según Torres (1996), la estructura de las guías es secuencial y graduada. Su objetivo es facilitar el manejo del tiempo en el aula, agilizar la tarea del profesor en la atención a todos los grados y promover el uso de los rincones de aprendizaje y la biblioteca escolar. Los libros de autoaprendizaje trabajan con un método inductivo dividido en actividades 
básicas, cuento pedagógico, actividad práctica y actividad de recuperación a evaluar en el control de progresos. Por último, se identifican falencias y se realizan aportes en la ficha de adaptación (Gómez, 2010).

No obstante, Barreto et al. (2015) resaltan que el afán por finalizar las unidades interfiere en el desarrollo de las secuencias didácticas y entorpece la enseñanza de la lectura y de la comprensión. Además, la falta de actualización de contenidos, la repetición de actividades y la inexistencia de material para las pruebas Saber son algunas de las quejas generales (Bernal-Pinzón, 2017).

En las guías de ciencias naturales, se analizó la ausencia de la temática de las especies no carismáticas, por lo que hace falta presentar actividades y proyectos de intervención local. Además hace falta dar uso ilustrativo y no decorativo de las imágenes del texto (Torres \& Rojas, 2017).

En lengua castellana, se debe priorizar la lectoescritura. Su aprendizaje no es óptimo debido a la falta de compromiso de los docentes, a la desactualización pedagógica y a la escasez de material didáctico (Reimers, 2003).

Schiefelbein (1993) y Colbert (2006) señalan que el currículo de EN propone en sus recursos la biblioteca, rincones de aprendizaje, mobiliario, centros de aprendizaje y un plano de la zona. Dentro de los materiales de bajo costo, Torres (1996) se refiere la ficha familiar, el calendario agrícola, al croquis y a la monografía veredal. Con respecto al recurso de capacitación, Schiefelbein (1993) indica que esta debe ser continua e incluir alianzas con universidades y visitas a escuelas demostrativas, a seminarios y a microcentros.

La Escuela Activa Urbana [EAU] clasifica sus recursos en convencionales y no convencionales, lo cual permite la promoción de investigaciones y la transversalidad de saberes en el aula. Entre los recursos se encuentran guías, carteles, libros, cuadernos viajeros, el medio natural y material didáctico elaborado por estudiantes, docentes o padres de familia. Esto lleva a que el docente replantee sus prácticas desde una mirada investigativa y mediadora entre el conocimiento y los estudiantes (Rojas, 2007; Schiefelbein, 1993; Villar 2010).

Las características, los recursos y los materiales predominantes derivan de una serie de métodos que el modelo acoge. Londoño (2002) prioriza el desarrollo de proyectos en la formación de la racionalidad a partir de la curiosidad, del cuestionamiento y de la supresión de prejuicios y asume el método global de Decroly en la enseñanza de la lectura. Un ejemplo representativo, según Cardoso (2003), es la cartilla La alegría de leer.

En ciencias naturales se recurre al método inductivo y experimental. Según Ríos y Cerquera (2013), los saberes de esta área se constituyen a partir de la observación y de la experimentación. Gómez (2010) complementa la afirmación anterior con el uso de procesos deductivos por descubrimiento de detalles que inducen a conclusiones generales para aumentar el interés, la motivación y la iniciativa en el aprendizaje.

Promover la participación de la comunidad hace parte del método de EN. Por esto, se establecen espacios escolares, culturales y recreativos, tales como el día de logros y las actividades del gobierno escolar (Torres, 1996). 
El éxito curricular está ligado a la calidad de la capacitación docente, que ha mejorado gracias al rol de las instituciones formadoras. González et al. (2015) mencionan las escuelas de Toca y de San Mateo - Boyacá-, en las que el $87 \%$ de los docentes de primaria son licenciados con especialización. Sin embargo, la problemática radica en la falta de compromiso social y pedagógico de los maestros. Según Torres (2015), el profesor debe actualizarse constantemente, aunque su labor se vea empañada por la falta de recursos, el olvido por parte del gobierno y la baja remuneración salarial. Por otra parte, Torres (1996) expone que es preciso focalizar la motivación y la innovación a partir del intercambio de experiencias en microcentros y del análisis crítico de sus propias prácticas con orientación de expertos. Como respuesta a esto existe un equipo departamental de multiplicadores con el objetivo de enseñar cómo implementar el modelo de acuerdo con las condiciones locales, al incorporar elementos culturales al currículo (Villar, 2010).

Un gran número de evaluaciones de EN colombiana se han realizado por medio de investigaciones sobre rendimiento, informes de los supervisores y de los coordinadores de microcentros y de visitas realizadas por expertos para identificar necesidades y falencias al innovar (Schiefelbein, 1993).

Los aspectos positivos de la evaluación fueron resaltados por la Unesco en 1985, por ser una experiencia que permite la educación cívica, la creatividad y el fortalecimiento de la autoestima. Organizaciones como Save the children y Unicef reúnen esfuerzos para llevar esta metodología a otros países de América Latina y el Caribe (Schiefelbein et al., 1996).

Finalmente, el estudio comparativo de la Unesco de 1998 concluye que Colombia fue el único país en el que la escuela rural obtuvo mejores puntajes que la urbana. En 2002 demostró que los resultados en matemáticas de contextos rurales solo fueron superados por Cuba (Colbert, 2006).

Segundo desplazamiento: métodos y relación con el sujeto

La metodología es un conjunto de técnicas e instrumentos directamente ligados a la práctica pedagógica en función de los objetivos trazados por el modelo. Para Gómez (2010), la EN trabaja una metodología individualizada y flexible, conectada con la vida cotidiana del estudiante y el principio de actividad reflejado principalmente en el juego como herramienta que simula la realidad y potencia el desarrollo del cuerpo, de la mente y de las habilidades sociales. La aplicación de proyectos favorece las relaciones interpersonales, el diálogo, la comunicación, el contacto con la naturaleza y el liderazgo (Flórez, 1995; López, 2007; Marcel, 2003).

En relación con las estrategias empleadas comúnmente, Rojas (2007) resalta las exposiciones, las mesas redondas, los foros, la narración de experiencias, las declamaciones y los trabajos de campo con la comunidad para potenciar habilidades comunicativas, oratorias e intelectuales y para formar gestores sociales. Sin embargo, la necesidad de mejorarlas es latente. Muestra de ello son las dificultades de los niños en la comprensión lectora, para lo que Barreto et al. (2015) y Sánchez et al. (2016) consideran necesario complementar su contenido con la inclusión de pruebas Saber que permitan la intertextualidad y la investigación en el aula. Así mismo, consideran necesario utilizar la lectura literal como un mecanismo didáctico para la resolución de problemas matemáticos, al aprovechar los 
procesos mentales que se realizan para establecer el significante, el significado y el sentido de las palabras.

Frente a la misma inquietud, Ávila et al. (2018) destacan la Institución Educativa Técnica Antonio Nariño de San Eduardo. Evidencian que algunos problemas en el desarrollo de las actividades están ligados a la lectura silábica, a la falta de atención y a la dificultad para argumentar y seguir instrucciones. Así, se desarrolló una propuesta didáctica basada en las relaciones horizontales, el trabajo entre pares, el juego y el uso de las TIC.

La habilidad lectora es indispensable en el proceso educativo, debido al uso de material de autoinstrucción. Ramírez, Portela y Amador (2010) afirman que una falencia detectada en las cartillas de educación física es la falta de claridad en las indicaciones y en el contenido, lo que entorpece la comprensión y exige pensar en estrategias enmarcadas en el conocimiento de sí mismo, del entorno vivo y físico, en el trabajo grupal y en las capacidades sociomotrices. Por otro lado, deben contar con ilustraciones explicativas y reforzar el trabajo manual -en los grados inferiores - y el componente competitivo - en grados superiores- (Ramírez, Ardila \& Montaño, 2013).

El abordaje de la enseñanza de las matemáticas requiere reconocer el uso de las TIC como una estrategia lúdica vanguardista. La aplicación de Scratch y de proyectos interactivos en el aprendizaje de las tablas de multiplicar es una experiencia vivencial, significativa y motivacional que se acopla al modelo de EN (Vivas et al., 2016).

Según Ramírez (2008), el aprendizaje del inglés es catalogado como difícil y de poco interés. Por ello es importante recurrir al enfoque comunicativo, centrar la atención en el estudiante y replantear la organización de grupos de trabajo. Para Murcia (2014), se debe comprender el aprendizaje colaborativo como un esquema de inteligencia social en el cumplimiento de objetivos compartidos.

Para Suárez et al. (2015) es pertinente considerar los espacios de aprendizaje. Dentro de ellos se destaca el laboratorio, la sala de informática, los escenarios deportivos y los proyectos productivos. Torres (1996) resalta la huerta, el jardín y el medio natural, el aire libre, las actividades grupales y las excursiones, las cuales, de acuerdo Zubiria (2019b) contribuyen al desarrollo de la personalidad y al aprendizaje significativo.

La calidad de los espacios de aprendizaje debe ser consecuente con el éxito esperado. A pesar de ello, la infraestructura de las escuelas rurales - como las del municipio de San Mateo, Boyacá- es precaria. El 66\% de las escuelas presenta agrietamientos y falta de servicios sanitarios y de campos deportivos (González et al., 2015).

Un aspecto importante del modelo de EN es que garantiza la participación escolar (Ríos, 2013), evidenciada en el gobierno estudiantil y en la conformación de comités (Villota, 2011), los cuales son elegidos a través del voto y están conformados por el presidente, el vicepresidente y el monitor de niveles, según lo hacen ver Torres (1996) y Villar (2010). Otro de los niveles que muestran la participación y ejercicio de la democracia es el uso de instrumentos como el autocontrol y el buzón de sugerencias (Psacharopoulos et al., 1996). Por esto se dio el reconocimiento Emprendedores por la paz, realizado por Ashoka en 2008 por haber logrado un cambio social hacia la paz en su componente democrático (FEN, 2019a). 


\section{Tercer desplazamiento: Escuela Activa como experiencia}

Según Rico y Spirko (2008), el Gimnasio Moderno fue la primera institución en Colombia que aplicó la pedagogía activa, como iniciativa de Agustín Nieto Caballero y de diecisiete jóvenes más para educar en autonomía, el liderazgo, el patriotismo y el emprendimiento social. Londoño (2002) centra la innovación en la disciplina de la confianza y en el respeto por la singularidad, plasmados en el proyecto democrático República escolar, enfocado en la responsabilidad, la justicia y el respeto.

Las escuelas normales son pieza fundamental en la apropiación del discurso activo por su naturaleza formadora. Según Londoño (2002), la perspectiva pedagógica de Decroly fue dominante en esta tarea y luego sustituida por la de Dewey, que está basada en la actividad pedagógica, en la investigación y en la democracia. Estas instituciones son catalogadas por Ríos (2013) como centros de indagación y de especialización docente y científica, gracias a su posición predominante en la expansión del conocimiento y de la pedagogía moderna con los ensayos y reflexiones en torno a la enseñanza en la EN.

El programa de EN surge como respuesta a las inconformidades de los docentes frente a la Escuela Unitaria. Ellos consideraban que era una estrategia del gobierno para recortar personal y sobrecargar de trabajo al maestro con la elaboración de fichas individualizadas (Colbert, 2006).

Gómez (2010) formula que, pese a la noción de esperanza frente a las pretensiones del modelo de la EN en Colombia, este dista mucho de las expectativas. Sus bajos costos se relacionan con la baja calidad, razón por la cual es necesario emprender procesos de investigación y de evaluación del modelo. La lucha frente al abandono gubernamental, la pobreza y la falta de oportunidades en las comunidades rurales del país obligan a maestros, padres y estudiantes a buscar medidas para solventar los problemas del contexto - tal es el caso de la escuela Hojas Anchas del departamento de Caldas- (Flórez, 1995).

Una experiencia de transformación es la escuela El Rosal de Cómbita -Boyacá-, como resultado del apoyo de la comunidad y de la FEN en el replanteamiento del plan de estudios, de la promoción de escuela de padres, del cooperativismo y del gobierno escolar, lo cual convirtió a la institución en escuela demostrativa (Cuadros, 2015).

Es pertinente aplicar elementos de la metodología activa para analizar limitaciones asociadas a la desmotivación y a la falta de pensamiento crítico, de autonomía y de apropiación del conocimiento en los procesos educativos de la educación superior. Sin embargo, los métodos de EN no podrían aplicarse en su totalidad, pero actuarían como una posibilidad para proponer nuevas estrategias pedagógicas (Redondo et al., 2015).

Villota (2011) menciona que la FEN emprende un nuevo proyecto denominado Círculos de aprendizaje, con apoyo de organizaciones internacionales, de la Universidad de Nariño y del MEN, los cuales asumieron el reto de mitigar el impacto social de la guerra. El gobierno escolar y de padres, la organización comunitaria y el rescate del diálogo hacen parte de la estrategia para sanar y fortalecer el tejido social y la cultura de paz (FEN, 2019a)

Los fundamentos de la EA también se materializaron en el modelo de EAU. Ramírez (2008) habla de la participación de la Fundación Luker, la Secretaría de Educación de Manizales, empresarios por la educación, la Fundación Ford y la Fundación Génesis, lo 
que consolidó la innovación de competencias laborales, argumentativas, comunicativas y de emprendimiento en la educación media, la mejora en los resultados de las pruebas Saber y la participación de los padres en la formación académica de sus hijos (González \& Izasa, 2007).

\section{Conclusión}

Los primeros discursos de EA hacen énfasis en la labor eugenésica de la educación para mejorar las condiciones sociales, culturales y económicas, a partir de la relación entre actividad, idealización de la raza, civilización, progreso, evolución biológica, psicología genética, pedagogía científica y mediación social. Por esta razón, el modelo ha llegado a comunidades vulnerables y marginadas, y se ha adaptado a las necesidades del contexto. Este discurso se transforma según intereses culturales, sociales y políticos, traducidos en el avance social y económico nacional, enmarcados en los principios de cobertura y calidad como símbolo de igualdad. Se prioriza la construcción de habilidades sociales, laborales, cívicas y democráticas, que actualmente responden a la necesidad de construir tejido social y cultura de paz.

En cuanto a las prácticas, se destaca el uso de guías y de material de autoaprendizaje, la flexibilidad y contextualización curricular, el desarrollo de proyectos, el contacto con el medio, el trabajo colaborativo, el método global, el método científico-experimental, el aprendizaje social y la educación democrática. Los fundamentos de EA se manifestaron principalmente en las experiencias del Gimnasio Moderno, de las escuelas normales, de la escuela unitariamultigrado rural, del modelo pedagógico de la EN, de los círculos de aprendizaje de la FEN y de EAU.

Se evidencia mayor productividad en la Universidad Nacional frente a los discursos de la EN. La UPTC enfatiza los procesos investigativos de prácticas y experiencias. La Sociedad Colombiana de Pedagogía presenta amplia participación en el análisis de los efectos de esta metodología en procesos cognitivos y sociales. Así mismo se observa alta incidencia del programa de EN en las manifestaciones activas directamente relacionadas con el currículo, con la pedagogía y con la educación. Por el contrario, a nivel general, la categoría con menor interés en los artículos fue la historia de la EA.

El balance resalta enunciados que se centran en los componentes teórico, curricular y metodológico, en cuanto a sus aportes en procesos cognitivos y sociales. Además, se evidencian vacíos y sugerencias investigativas en torno a la inclusión de la informática y el bilingüismo, así como al énfasis en la promoción de la capacitación docente que tenga como eje central el compromiso social, pedagógico y la vocación, para promover la innovación y la motivación desde los microcentros y el análisis crítico de la práctica.

La cantidad de críticas frente a las guías de aprendizaje es clara en el estado del arte. Sin embargo, los aportes sobre la actualización de elementos concretos es casi nula, razón por la que varios autores hacen referencia a la necesidad de realizar investigaciones que permitan mejorar futuras ediciones de las cartillas de autoaprendizaje, con la incorporación de material de apoyo para las pruebas Saber y con la ampliación de contenidos y didácticas, teniendo en cuenta los intereses de padres, de estudiantes y de desertores. De igual forma, es necesario estudiar la funcionalidad y el impacto del modelo en el aumento de la calidad respecto a los retos actuales. 


\section{Referencias}

Ávila, H., Jiménez, R., \& Torres, J. (2018). Comprensión lectora en estudiantes de primero a tercero de Escuela Nueva. Educación y Ciencia, (21), 93-107.

Barragán, B. (2008). María Montessori: Los límites de la escuela activa, una lectura arqueológica. Nodos y Nudos, 3(25), 92-101.

Barrantes, R. (2001). Las innovaciones educativas: escenarios y discursos de una década en Colombia. Magisterio, 1-38.

Barreto, M., Leguizamón, M., \& Torres, J. (2015). La comprensión lectora a través de las guías de aprendizaje en la Escuela Nueva. Educación y Ciencia, (19), 163-172.

Bernal-Pinzón, M. (2017). ¿Qué escriben los niños? Una mirada desde el modelo Escuela Nueva. Revista de Investigación, Desarrollo e Innovación, 7(2), 255-268. https://doi. org/10.19053/20278306.v7.n2.2017.6069

Calvache, J. (2003). La Escuela Nueva y los conceptos básicos de la educación en el pensamiento de John Dewey: Una aproximación teórica. Revista Historia de la Educación Latinoamericana, 5, 107-126.

Cardoso, N. (2003). La Escuela Activa y el texto Alegría de leer en Colombia. Revista Historia de la Educación Latinoamericana, 5, 127-138.

Colbert, V. (2006). Mejorar la calidad de la educación en escuelas de escasos recursos. El caso de la Escuela Nueva en Colombia. Revista Colombiana de Educación, (51), 185-212.

Cuadros, J. (2015). Escuela Nueva: Una estrategia pedagógica en la Institución Educativa Agropecuaria Santa Bárbara. Revista Historia de la Educación Colombiana, 18(18), 239262.

Decreto 1490 de 1990. Diario oficial número 39461, Bogotá, Colombia, 11 de julio de 1990.

Flórez, R. (1995). La dimensión pedagógica, formación y Escuela Nueva en Colombia. Educación y pedagogía, (14-15), 197-219.

Fundación Escuela Nueva. (2019a). Cooperative Learning in Escuela Nueva Activa. Magisterio. https://www.magisterio.com.co/articulo/cooperative-learning-escuela-nueva-activa

Fundación Escuela Nueva. (2019b). La educación para la paz que promueve la Fundación Escuela Nueva Volvamos a la Gente.Magisterio. https://www.magisterio.com.co/articulo/ la-educacion-para-la-paz-que-promueve-la-fundacion-escuela-nueva-volvamos-la-gente

García, N., \& Rojas, S. (2015). La enseñanza de la lectura en Colombia: enfoques pedagógicos, métodos, políticas y textos escolares en las tres últimas décadas del siglo XX. Pedagogía y Saberes, (42), 43-60. https://doi.org/10.17227/01212494.42pys43.60

Gómez, V. (2010). Visión crítica sobre la Escuela Nueva en Colombia. Revista de Educación y Pedagogía, 7(14-15), 280-306.

González, A., \& Isaza, S. (2007). Escuela Activa Urbana. Plumilla Educativa, 4(1), 7-10. https://doi.org/10.30554/plumillaedu.4.598.2007

González, A., Regalado, M., \& Jiménez, A. (2015). La pedagogía activa con metodología 
escuela nueva en Boyacá: el caso de dos municipios. Quaestiones Disputatae: temas en debate, 8(16), 83-101.

Guerrero, A. (2011). Don Jorge Buendía, el impulsor de la Escuela Activa en el sur de Colombia durante el siglo XX. Historia de la Educación Colombiana, (14), 147-168.

Imbachí, M. (2013). La Escuela Nueva y las competencias lectoras desarrolladas en el área de lengua castellana. Revista Digital de Ciencias Aplicadas al Deporte, 5(11), 46-53.

Jiménez, Á. (2009). La escuela nueva y los espacios para educar. Educación y Pedagogía, 21(54), 103-125.

Jiménez, A. (2018). Primer congreso pedagógico nacional colombiano de 1917. Pedagogía $y$ Saberes, (48), 153-161.

León, G. (2002). Imposición de modelos pedagógicos en Colombia - Siglo XX. Estudios latinoamericanos, (10-11), 21-32.

Londoño, C. (2002). El pragmatismo de Dewey y la escuela nueva en Colombia. Revista Historia de la Educación Colombiana, 5(5), 143-169.

López, M. (2007). Aportes de la pedagogía activa a la educación. Plumilla Educativa, 4(1), 33-42. https://doi.org/10.30554/plumillaedu.4.605.2007

Marcel, Á. (2003). Don Agustín Nieto Caballero andante de la educación. Revista Historia de la Educación Latinoamericana, 5, 85-106.

Mejía, M. (2015). La educación popular en el siglo XXI. Una resistencia intercultural desde el sur y desde abajo. Praxis \& Saber, 6(12), 97-128. https://doi.org/10.19053/22160159.3765

Murcia, N. (2014). Comunidades de práctica: Huellas en el currículo de la Escuela Activa Urbana en Manizales. Revista Criterios, 21(1), 15-40.

Pineda, N. (2014). Reflexiones sobre la labor docente en escuelas rurales que implementan la metodología escuela nueva. Quaestiones Disputatae: Temas en Debate, 7(15), 33-50.

Psacharopoulos, G., Rojas, C., \& Vélez, E. (1996). Evaluación de resultados en la Escuela Nueva de Colombia ¿Es el multigrado la respuesta?. Revista Colombiana de Educación, (32), 1-14.

Presidencia de la república - Colciencias. (1995). Colombia: Al filo de la oportunidad. Misión Ciencia, Educación y Desarrollo. Tomo 1. http://www.plandecenal.edu.co/cms/ media/herramientas/colombia_al_filo_de_la_oportunidad.pdf

Ramírez, C., Ardila, W., \& Montaño, N. (2013). Tareas motrices. Apuesta comprensiva para su diseño metodológico en Escuela Nueva. Impetus, 7(1), 1-6.

Ramírez, C., Portela, H., \& Amador, L. (2010). Actitud y valores en la clase de educación física. Un caso desde el modelo Escuela Nueva. Revista de Investigaciones, (16), 38-45.

Ramírez, O. (2008). The English Language Learning inside the Escuela Activa Urbana Model in a public School a study of sixth Graders. Profile, 9(1), 47-62.

Ramos, D., \& Romanowski, J. (2014). Estudos de revisão: implicações conceituais e metodológicas. Revista Diálogo Educacional, 14(41), 165-189. https://doi.org/10.7213/ dialogo.educ.14.041.DS08 
Redondo, K., Redondo, C., \& Martínez, V. (2015). Aportes del proyecto 'Escuela Nueva' a la educación universitaria en Colombia. Revista Ciencias Biomédicas, 6(2), 390-393.

Reimers, F. (2003). Aprender a leer y a convivir en las escuelas rurales de Colombia. Pedagogía y Saberes, (18), 5-20.

Rico, F., \& Spirko, J. (2008). Crónica sobre el Gimnansio Moderno. Revista Educación y Humanismo, (14), 179-188.

Ríos, R. (2013). Escuela Nueva y saber pedagógico en Colombia: apropiación, modernidad y métodos de enseñanza. Primera mitad del siglo XIX. Historia y sociedad, (24), 79-107.

Ríos, R., \& Cerquera, M. (2013). La modernización de los contenidos y métodos de enseñanza: Reflexiones sobre la Escuela Nueva en Colombia. Revista Historia de la Educación Latinoamericana, 16(22), 157-172. https://doi.org/10.19053/01227238.2693

Rojas, Y. (2007). Escuela activa urbana. Un proyecto sostenible. Plumilla Educativa, 4(1), 23-26. https://doi.org/10.30554/plumillaedu.4.602.2007

Ruiz, L., \& Pineda, J. (2007). Hacia una fundamentación epistemológica del modelo de pedagogía activa. Plumilla Educativa, 4(1), 48-51. https://doi.org/10.30554/ plumillaedu.4.607.2007

Runge, A., \& Muñoz, D. (2011). Actividad vs. agitación en el pensamiento de Luis López de Mesa: relaciones entre pedagogía y eugenesia en la Colombia de principios del siglo XX. Revista Colombiana de Educación, (61), 21-51.

Sáenz, J. (1997). De lo biológico a lo social. Saber pedagógico y educación pública en Colombia. Revista Educación y Ciudad, (4), 120-125.

Sánchez, V., Amado, Y., \& Bolívar, A.(2016). Enseñanza de lectura literal en resolución de problemas matemáticos en Escuela Nueva. Educación y Ciencia, (19), 23-37.

Schiefelbein, E. (1993). El modelo Escuela Nueva: Intentos de descripción. En En busca de la escuela del siglo XXI ¿ Puede darnos la pista la Escuela Nueva en Colombia? (pp. 18-38) Unesco-Unicef.

Schiefelbein, E., Vera, R., Aranda, H., Vargas, Z., \& Corco, V. (1996). En busca de la escuela del siglo XXI ¿Puede darnos la pista la Escuela Nueva de Colombia?. Revista Colombiana de Educación, (32), 1-46.

Suárez, D., Liz, A., \& Parra, C. (2015). Construyendo tejido social desde la Escuela Nueva en Colombia. Un estudio de caso. Revista Cientifica General José María Córdoba, 13(15), 195-229.

Torres, N. (2015). Agustín Nieto Caballero: Pensamiento Pedagógico y aportes a Escuela Nueva. Revista Unimar, 33(1), 57-73.

Torres, N., \& Rojas, M. (2017). Enseñanza de especies no carismáticas invertebradas en el contexto de la Escuela Nueva: Un análisis desde los libros de texto. Bio-grafía, 10(19), 85-100. https://doi.org/10.17227/bio-grafia.vol.10.num19-7223

Torres, R. (1996). Alternativas dentro de la educación formal: El programa Escuela Nueva en Colombia. Revista Colombiana de Educación, (32), 1-12. 
Urrea, S. (2017). Las agencias internacionales, su papel en la creación y expansión del modelo Escuela Nueva. Ágora USB, 17(1), 95-104.

Villar, R. (2010). El programa de Escuela Nueva en Colombia. Revista de Educación y Pedagogía, (14-15), 357-382.

Villota, Ó. (2011). La vieja escuela nueva. Unimar, (58), 47-55.

Vivas, C., Murillo, Z., \& Cristancho, J. (2016). Scratch. Estrategia didáctica para el aprendizaje de las tablas de multiplicar en Escuela Nueva. Educación y Ciencia, (20), 43-58.

Zubiría de, J. (2019a). ¿Favorece la Escuela Nueva el desarrollo del interés por el conocimiento? Magisterio, 12-15.

Zubiría de, J. (2019b). 4 aportes de la Escuela Nueva. Magisterio, 114-117.

Zubiría de, J. (2019c). Escuela Nueva: ¿Desarrolla el activismo la flexibilidad?. Magisterio, 129-132. 